\title{
Formación ciudadana y participación política en jóvenes de la Universidad del Bío-Bío, Chile ${ }^{1}$
}

Civic education and political participation among youth at Universidad del Bio- Bio, Chile

Recibido: 22 de septiembre de 2014 - Revisado: 22 de febrero de 2015 - Aceptado: 10 de abril de 2015

\author{
Cristian Orellana Fonseca ${ }^{2}$ - Bruno Bivort Urrutia ${ }^{3}$ \\ Soledad Martínez Labrín ${ }^{3}$ - Fernando Farías Olavarría ${ }^{3}$ \\ Héctor Cárcamo Vásquez ${ }^{4}$ - Enrique Blanco Hadi ${ }^{5}$ \\ Gabriel Pérez Díaz
}

\section{Resumen}

Los resultados de este artículo son producto de una investigación mayor sobre participación política. En él se analizan los discursos de jóvenes universitarios respecto de la formación ciudadana recibida en la escuela, en su vínculo con la participación política. Se realizaron tres focus group con estudiantes de primer año de carreras de la Universidad del Bío-Bío, Chile. Los resultados apuntan que la visión de los jóvenes sobre la formación recibida es más bien crítica. Por un lado, se identifica la necesidad de una formación ciudadana, que permite abordar la complejidad de la actividad política. Por otro, se constata que la formación escolar no responde a esta necesidad, puesto que se califica como deficiente y sesgada. Para los estudiantes, la educación debe asumir un rol politizador, entregando insumos para incidir en el cambio social.

\section{Palabras clave}

Formación ciudadana, participación política, jóvenes.

\begin{abstract}
The results of this paper are the product of a broader research on political participation. The expressions of young university students are there analyzed about civic education received at school related to political participation. Three focus groups were held with freshmen at Universidad del Bio-Bio, Chile. The results show that the vision of young people about the formation received is rather critical. On the one hand, the need for civic education is identified as that which allows to address the complexity of political activity. Secondly, it is found that school education does not respond to this need, since it is qualified as poor and biased. For students, education must play a politicizing role, providing inputs to influence social change.
\end{abstract}

\section{Keywords}

Civic education, political participation, youth.

\footnotetext{
${ }^{1}$ Artículo resultado del proyecto de investigación: "Discursos y prácticas de ciudadanía y jóvenes universitarios", financiado por la Universidad del BíoBío, Concepción, Chile.

${ }^{2}$ Sociólogo. Profesor de Historia y Geografia, magíster en Investigación Social y Desarrollo. Académico de la Facultad de Educación y Humanidades, Universidad del Bío-Bío. Integrante del Observatorio Prospectivo Educación y Desarrollo Regional y del Programa de Derechos Humanos de la Universidad del Bío-Bío, Concepción, Chile.

Correo electrónico:

corellana@ubiobio.cl

${ }^{3}$ Integrantes del Grupo de Investigación en Género, Ciudadanía y Equidad (GECIEQ) de la Dirección General de Investigación y Desarrollo de la Universidad del Bío-Bío, Concepción, Chile.

Correos electrónicos:

bbivort@ubiobio.cl,cmartine@ubiobio.cl, ffarias@ubiobio.cl.

${ }^{4}$ Sociólogo. Magíster en Investigación Social y Desarrollo. Doctor en Antropología Social por la Universidad Complutense de Madrid. Académico de la Facultad de Educación y Humanidades, Universidad del BíoBío, Concepción, Chile.

Correo electrónico:

hcarcamo@ubiobio.cl

${ }^{5}$ Doctor en Ciencias de la Educación, profesor de Filosofía. Académico del Departamento de Educación, Facultad de Educación y Humanidades, Universidad del Bío-Bío, Concepción, Chile.

Correo electrónico:

eblanco@ubiobio.cl

${ }^{6}$ Licenciado en Sociología, Universidad de Concepción, Concepción, Chile. Correo electrónico: gabrielperez@udec.cl

Para citar este artículo: Orellana, C., Bivort, B., Martínez, S., Farías, F., Cárcamo, H., Blanco, E., \& Pérez, G. (2015). Formación ciudadana y participación política en jóvenes de la Universidad del Bío-Bío, Chile. Revista Civilizar Ciencias Sociales Humanas, 15(28), 119-134.
} 


\section{Introducción}

En este artículo se analizan los discursos de jóvenes universitarios respecto de la formación ciudadana recibida en la escuela, en su vínculo con la participación política. Para ello, se revisa en primera instancia, las conceptualizaciones que se realizan desde la academia sobre la participación política juvenil, destacando la distinción política/político como un instrumento útil operativamente para el análisis de los datos.

Se pasa revista a la participación electoral de las últimas décadas en Chile, como una forma de contextualizar en la historia la problematización sobre la "baja participación". Luego se revisa el concepto de ciudadanía como una forma de entender la relevancia de la formación ciudadana, a la vez que se establece el nexo entre esta participación política. Finalmente, se aborda el estado de la formación ciudadana en el currículo escolar.

Con este marco de referencia, se analizan los datos, que están presentados con las siguientes categorías: necesidad de la formación ciudadana, formación ciudadana deficiente, formación ciudadana sesgada, rol politizador de la formación ciudadana y vínculo entre formación ciudadana y participación política. Las conclusiones plantean los desafíos tanto para la realización de la formación ciudadana en el aula, como para su ámbito administrativo, local y estatal.

\section{Participación política juvenil: la política y lo político}

En nuestro país, autores como Baño (1985), Zarzuri (2005) y Valenzuela (2007) visualizan una importante participación política en el sector juvenil, gracias a una concepción amplia, que no circunscribe el ámbito de participación al Estado y sus redes institucionales de administración del orden. Podemos encontrar un sustento teórico coherente para esta postura en estudiosos como Arditi (1995) y Retamozo (2009).
De acuerdo con Arditi (1995), Schmitt se propone generar un criterio para distinguir los fenómenos políticos de aquellos que surgen de otras esferas de actividad. Para ello sugiere entender lo político a través de una oposición basal: la distinción entre amigo y enemigo. El momento de lo político, entonces, se determinará por la intensidad de la separación en virtud de la cual se construyen las asociaciones y disociaciones decisivas.

Arditi (1995) rescata de esta definición, que el conflicto es una dimensión constitutiva de lo político, es decir, que lo político designa una forma de enfrentamiento y no el contenido sustantivo del mismo. Por tanto, lo político no puede convertirse en un ámbito central por sí solo, puesto que carece de preocupación propia, y porque potencialmente es capaz de englobar a todas las preocupaciones sociales. Así, todo es politizable, lo que no equivale a decir que todo es político.

Agrega que no es relevante que los reagrupamientos aparezcan como partidos políticos, o si los conflictos ocurren o no dentro del espacio estatal, o si se dirigen a controlar dicho espacio. Lo político "no está supeditado a lo que la sociedad reconoce formalmente como el campo de la política" (Arditi, 1995, p. 8). Es válido por ende, hablar de un desbordamiento de la política más allá del Estado o del sistema político. Aunque las referencias a lo político no implican una disolución de la esfera formal de la política.

Por otra parte, para Retamozo (2009) lo político corresponde al momento instituyente del orden social, mientras que la política corresponde a la administración de lo instituido. Los términos de esta distinción no se identifican con sujetos particulares, ni tampoco señalan momentos cronológicos. En su lugar, se utilizan como categorías teóricas que describen sistemas de interacciones y la consecuente formación de relaciones de poder, reconociendo en ellas, características, funciones y racionalidades dentro de los fenómenos considerados como políticos. 
Así, Retamozo (2009) lleva a cabo la construcción de un esquema conceptual pertinente para el estudio de los fenómenos políticos. Para ello recurre al rescate de varios autores de teoría política, entre ellos, Schmitt, Arendt, Lefort, Laclau y Ranciére. Del primero recupera el conflicto como condición de posibilidad de lo político y el hecho de que este supone un momento simbólico fundacional, donde se produce un acontecimiento social, potencialmente abierto y contingente.

Este momento es donde se efectúa la construcción de un antagonismo, lo que corresponde a un acto performativo. A su vez, esto va ligado a una producción de imaginarios sociales (Castoriadis, 2007, citado en Retamozo, 2009) capaces de pensar otros órdenes sociales posibles. En términos de Laclau (2000) sería la producción de discursos articuladores primordiales para desnaturalizar el orden hegemónico, o en otras palabras, lo instituido (citado en Retamozo, 2009).

El momento de lo político, lo instituyente, no puede perpetuarse, por lo que debe dar paso a la política, la administración de lo instituido. Retamozo (2009) explica que, el momento de la política supone la estabilización de la contingencia y por tanto, el encauzamiento del conflicto. Mientras que Schmitt indica que esto se logra externalizando el conflicto al ámbito internacional y/o a través de la policía en el ámbito interno. Arendt (1997) busca preservar el espacio de contingencia, desde el marco de una democracia deliberativa. Mientras que para el primero el conflicto constituye un peligro a la integración, para la segunda, permite una reconfiguración del orden social. Así, se establecen los contornos de la distinción: un momento de la institución del orden social: lo político, y uno donde se resuelven asuntos comunes, donde se administra y gestiona esa ordenación: la política (citado en Retamozo, 2009).

Ahora, dentro de las visiones que postulan la existencia de múltiples modos de par- ticipación política en jóvenes, se sostiene que estos varían en sus características y grados de institucionalización, según presenten un mayor o menor influjo adulto (Serna, 2000) y en consecuencia, adoptando formas diversas: desde organizaciones hasta colectivos (Garcés, 2010; Zarzuri, 2005).

Tenemos que, fuera de la participación política institucional y de la influencia adulta, se aprecia una presencia relevante de colectivos políticos (Acosta \& Garcés, 2010; Cárdenas, Parra, Picón, Pineda, \& Rojas, 2007; Navarrete, 2008; Reguillo, 2000; Sandoval \& Hatibovic, 2010; Valenzuela, 2007). Estas conformaciones suponen el planteamiento por parte de los jóvenes, de expresiones de organización política contrapuestas a la lógica representativa tradicional.

Tales características, ubican a dichas formas de participación política en un espacio de transición de la política a lo político, en lo que Retamozo (2009) con base en Laclau, denomina el recuerdo de la contingencia. Esto es, el cuestionamiento del orden social naturalizado, a través del conocimiento de la manera en que este se construyó en un comienzo. Esto da paso a un proceso de antagonización frente a la forma tradicional de hacer política, lo que se traduce en la articulación de nuevas subjetividades, que se expresan, por ejemplo, en la formación de colectivos que, independiente de sus contenidos sustantivos, comparten, por razones que ya explicamos, el adjetivo de "político".

\section{Sobre la participación política electoral en Chile}

Respecto a este punto, la mayor inscripción electoral en la historia del país fue en el plebiscito de 1988 (donde se votaba la continuidad o no de la dictadura cívico-militar), llegando al $97 \%$, allí los jóvenes representaron el $36 \%$ de las personas inscritas. En adelante, se experimenta un sostenido descenso, como lo muestra la elección parlamentaria de 1997, 
donde los jóvenes inscritos no alcanzaron al 20 \% (Fernández, 2000).

En 2003, alrededor de un $73 \%$ de los jóvenes no se encontraba inscrito en los registros electorales (Instituto Nacional de la Juventud -INJUV, 2003). En 2010, este porcentaje se eleva al 78,7 \% (INJUV, 2010). Dicha situación fue vista como un fenómeno muy preocupante (Navia, 2004), ya que cuestionaba la legitimidad y representación del sistema democrático en Chile. Para Urrutia (2004) la preocupación frente a la "baja participación política juvenil" refleja la manera en que opera el discurso dominante sobre la política.

$Y$ es que la democracia representativa de corte liberal se ha naturalizado con el tiempo, al punto de aparecer como la única configuración política posible (Rajland, 2003), hecho cuestionado con base en las investigaciones ya citadas, que dan cuenta de la existencia de otras lógicas de organización política. Además, cabe señalar que de acuerdo con Urresti (2000), atribuir el desencanto y despolitización a una cuestión voluntaria de un actor colectivo, como la juventud, es un procedimiento conceptual incorrecto.

En su lugar, corresponde comprender dichos actores en su relación con la situación histórica y social que les toca vivir. En esta línea, tenemos que el plebiscito de 1988, significó para los jóvenes que participaron de él, una lucha pacífica por sus ideales, y por tanto, un modo de rechazar la violencia de la dictadura, vivida cotidianamente. Fue entendido como una instancia capaz de generar un cambio social profundo, cambio que dependía solo de la opción que tomaran los ciudadanos (Rojas, 2006), es decir, una estrategia enraizada en el ámbito de lo político.

Veinte años después de este hito, la aprobación de la ley de inscripción automática y voto voluntario en 2011, pretende responder a la problematización sobre la participación po- lítica electoral "deficiente" de los jóvenes, lo que se puede leer como un intento de establecer formas que logren la internalización del modelo adulto en los jóvenes, de modo que cumplan normas que aseguran la legitimación del sistema de representación política (Valenzuela, 2007), dejando de lado la complejidad y multidisciplinariedad que demanda el abordaje del ejercicio de la ciudadanía (Mardones, 2012).

$\mathrm{Su}$ inefectividad resulta evidente en términos de los propios objetivos planteados. Este fracaso, a nuestro juicio, reside en que la comparación entre generaciones, dejó de lado elementos propios del contexto social, económico y cultural en que se produce la disminución de la participación electoral, que como vimos en el principio, se caracteriza entre otros aspectos, por la participación política juvenil heterogénea. Así la alta participación electoral, que tuvo la juventud en el periodo dictatorial chileno significó una estrategia para transformar el orden social (función instituyente), en la actualidad se inserta en el plano de la política, en la administración de lo instituido, desde una lógica política que evidencia no ser compartida mayoritariamente.

\section{Acerca del concepto de ciudadanía}

Antes de problematizar las ideas respecto a la formación ciudadana y su vínculo con la participación política, creemos necesario abordar el concepto de ciudadanía como elemento basal. En tal sentido, si realizamos una mirada retrospectiva, podemos afirmar que la ciudadanía (a partir de la Segunda Guerra Mundial) fue asumida como una categoría ligada esencialmente con la dimensión jurídica (jus solis/jus sanguinis), estableciéndose así una asociación naturalizada entre la nacionalidad y la ciudadanía (Domingo, 2001).

De este modo, la consanguinidad y el lugar de nacimiento se constituyen en los criterios que enarbolan los Estados-naciones para la defensa de los derechos de "sus ciudadanos", tal como lo 
planteó Marshall (1998). Tiempo ha pasado, y la ciudadanía comienza a vincularse más que con la noción de democracia representativa, con la de "democracia real", situación que implica pasar de una idea de ciudadanía pasiva estipulada en la ley a través de los derechos constitucionalmente definidos. Por ejemplo, mediante la reproducción de modelos electorales poco inclusivos cuya justificación se encuentra en la necesidad de mantener altos niveles de gobernabilidad en los países (por ejemplo el caso chileno), a una ciudadanía entendida en términos activos, que de acuerdo con Cortina (2001), conlleva intervenir en los asuntos públicos.

Este enfoque, presenta a la ciudadanía como una categoría flexible, dinámica y contextualizada en un espacio en el que tienen cabida diversas identidades; y la remite a un proceso con un gran potencial transformador en el que es posible reconocer tanto los derechos reales existentes (dimensión objetiva), como las formas de acercarse a dichos derechos (dimensión subjetiva). Cuando se conjuga una concepción flexible de ciudadanía y la existencia de diferentes dimensiones de la misma, se sientan las bases para la búsqueda y fijación de nuevos derechos.

En este sentido, la formación en ciudadanía es tarea fundamental, sobre todo en lo que respecta a la dimensión subjetiva. Desde esta perspectiva, las cualidades y actitudes de los ciudadanos son cruciales para la estabilidad de las democracias, lo que constituye un motivo más para subrayar la importancia de su formación en y desde la escuela, en articulación con los demás mundos vividos por los jóvenes (Díaz, 1996).

En relación con este último punto, algunos investigadores (Vargas, 2005; Malone \& Julian, 2005) problematizan el vínculo entre participación política y "conocimiento cívico". Para Vargas (2005), una de las variables que aportan mayor explicación sobre la participación política son las actitudes políticas y, dentro de estas, el conocimiento político. Mientras que para Malone y Julian (2005) la falta de participación institucional de los jóvenes se encuentra unida a que no cuentan con conocimientos cívicos, es decir, conocimientos sobre el gobierno, el sistema político, etc., ya que en la medida en que aumenta la capacidad cívica de los jóvenes, se incrementará su participación política.

\section{La formación ciudadana en el currículo escolar}

Investigaciones como las de Mardones (2012), Muñoz, Vásquez y Reyes (2010) y Muñoz y Torres (2014), que ligan formación ciudadana y participación política, hacen un balance negativo en materia de formación ciudadana para nuestro país. Igualmente, estudios como el de la Asociación Internacional para la Evaluación del Logro Educativo (IEA, por su sigla en inglés) (2010) dan cuenta de deficientes resultados obtenidos por nuestro país en la medición de "conocimiento cívico", ubicándonos en las últimas posiciones.

La preocupación desde la institucionalidad por la baja participación electoral del segmento joven, se refleja en publicaciones de apoyo a la formación ciudadana dirigida a profesores (Ministerio de Educación de la República de Chile -Mineduc, 2004), donde se pasa revista a la evolución que ha tenido esta desde inicios del siglo veinte hasta la reforma de los noventa, llevada a cabo por La Concertación ${ }^{1}$.

A principios del siglo veinte, con la incorporación de la asignatura de educación cívica en el currículo de los niveles primario y secundario, lo que se perseguía era la entrega de preceptos jurídicos, políticos y económicos, orientaciones que sirvieran para regir la conducta social según criterios morales que invocaban el bien público, lo que supone, tal como nos indica Castro-Gómez (2000) el modelamiento de un sujeto en específico, que respondiera a las necesidades del Estado-nación de la época. 
Este enfoque se mantuvo hasta la década de los setenta. La reforma educativa de la época, trajo consigo modificaciones sustantivas: la desaparición en la educación media de la educación cívica como asignatura, y su reemplazo por una introducción a la ciencia económica y política que se impartía en la asignatura de ciencias sociales, y la incorporación de la temática en las asignaturas de castellano, filosofía, matemáticas y ciencias naturales. Además, se potenció la organización estudiantil con la formación de los centros de alumnos y las agrupaciones de padres y apoderados que quisieran colaborar con la formación de sus hijos en corporaciones, mediante la realización de actividades de formación intelectual, cívica y social.

Esto terminó con la reforma de los ochenta, que generó un retorno a la situación anterior a la reforma de los setenta, es decir, a una asignatura de educación cívica y economía, dictándose educación cívica en $3^{\circ}$ año medio y economía en $4^{\circ}$ año medio. En la educación básica, el abordaje se realizaba dentro del área de historia y ciencias sociales.

La reforma de los noventa, enmarcada en el periodo de la "transición a la democracia" trata de reforzar e incorporar contenidos tendientes a que los estudiantes se familiarizaran con el régimen democrático, para distinguirlo de uno autoritario, apuntando a la vez, a desarrollar habilidades y actitudes encaminadas al fortalecimiento de una sociedad democrática. Así, en el año 1996 se introducen los objetivos fundamentales y contenidos mínimos que contempla la formación ciudadana en la actualidad.

Esta reforma estableció cambios primordiales, siendo el más significativo la extensión del concepto de "educación cívica", que se restringía a conocimientos sobre el Estado y sistema político, al de "formación ciudadana", que comprende conocimientos, habilidades y actitudes requeridas para la vida e institucionalidad democráticas y desarrolla temas vinculados a la historia política cercana como derechos huma- nos, autoritarismo, democracia, manejo crítico de la información, responsabilidad social, entre otros (Mineduc, 2004).

A partir de esta transformación conceptual se articulan los otros grandes cambios, que significaron: a) la asignación de objetivos y contenidos respecto de la formación ciudadana a las asignaturas de historia y ciencias sociales, lenguaje y comunicación, orientación y filosofía. Y b) el establecimiento de los objetivos fundamentales transversales (OFT), que explicitan los valores, actitudes y habilidades que se estima de relevancia para la democracia y la ciudadanía. Estos asignan la responsabilidad de crear oportunidades de aprendizaje para que los alumnos adquieran y desarrollen competencias para la ciudadanía, al profesor y la profesora, orientando moral y cognitivamente los aprendizajes particulares que se promueven desde las distintas asignaturas (Mineduc, 2004).

Con este cambio radical, desde la "educación cívica" de principios del siglo pasado al de la "formación ciudadana" de la actualidad, se distingue la intención -al menos en términos formales- de estimular en los estudiantes habilidades que les permitan entender la contingencia y la historicidad del orden social y político actual, naturalizado y objetivado en el Estado y sus instituciones, y no simplemente desarrollar una subjetividad proclive a cumplir con la reproducción y administración del mismo.

\section{Método}

Los resultados que se presentan en este artículo se alcanzaron en el marco de una investigación mayor sobre participación política. Esta investigación se compuso de una primera etapa de tipo cuantitativo, en la que los datos se obtuvieron a través de la aplicación de un cuestionario autoadministrado de preguntas cerradas. En una segunda etapa, de carácter cualitativo, se buscó la profundización de dichos datos, que fueron presentados como reactivo para la discusión en los focus group. 
Es importante destacar que el tema de la formación ciudadana no fue incluido como tópico de discusión por parte de los investigadores, sin embargo, emergió con fuerza desde los discursos de los participantes. La técnica del focus group fue seleccionada, puesto que resulta muy adecuada cuando el objetivo de una investigación requiere la recolección de información en profundidad sobre las necesidades, preocupaciones y percepciones de un colectivo social determinado, en este caso, jóvenes universitarios.

Se realizaron tres focus group con estudiantes de primer año de la Universidad del Bío-Bío, Chile. Uno con estudiantes de Pedagogía en Educación General Básica, otro con estudiantes de Trabajo Social y el tercero con estudiantes de Psicología, para así convocar diversas carreras que forman parte de la Facultad de Educación y Humanidades. El criterio de inclusión de los participantes fue la voluntariedad de la participación.

El análisis de los datos cualitativos se realizó con un modelo de reducción y categorización, con ayuda del software Atlas. ti 6.2. Cabe señalar que en la presentación de resultados se manejó el concepto de formación ciudadana, puesto que como ya aclaramos, su significado no se reduce a la mera transmisión de conocimientos, sino que incluye el desarrollo de habilidades y actitudes. Por lo mismo, ayuda a incluir términos como el de "educación política escolar", o "educación cívica", que son algunos de los que emplearon los estudiantes.

\section{Resultados}

A continuación se exponen los principales elementos que caracterizan los discursos de los estudiantes respecto de la formación ciudadana. Estos elementos fueron sistematizados y ordenados en cinco grandes categorías, que emergieron a partir de los datos. Más adelante, se describe cada una de las categorías con sus principales componentes.

\section{Necesidad de la formación ciudadana.}

La formación ciudadana la entienden los estudiantes como una necesidad en tanto supone, por un lado, una forma de subsanar una carencia formativa en el plano de lo político, ya sea a causa de un entorno familiar y/o cercano donde no se ha dado una socialización política significativa, como de una formación política deficiente en el ámbito escolar. Entonces, se ve la formación ciudadana como una manera de sobreponerse a esta carencia auto-observada, con el objeto de tener la información necesaria para participar políticamente. $\mathrm{Y}$ es interesante como aquí, se liga también la formación ciudadana con la participación, lazo que para los informantes resulta indispensable, lo que queda ilustrado en el siguiente extracto:

En lo personal, por mí, no participo por ignorancia. Como mencionaron antes, en mi familia, en mi entorno cercano no se vive, no se conversa de eso y en el futuro yo creo que ya tendría que empezar a investigar, a ver todos los puntos de vista para llegar a participar en algo [...] (comunicación personal, FG2, s.f.).

Por otro lado, la formación ciudadana se entiende como una necesidad para abordar la complejidad de la política, puesto que asumen que la actividad política demanda la posesión y el manejo de conocimientos diversos, no solo relacionados con el funcionamiento de las instituciones, sino también, por ejemplo, de tipo histórico. La ausencia de este saber supone falta de compromiso, y por ende, falta de participación política. Por ello, igualmente vuelve a surgir esta ligazón necesaria entre formación política y participación, que sirve para entender que los estudiantes tipifiquen la formación ciudadana como imprescindible: "No sé, eso encuentro yo, que la política es algo tan complicado, tan complicado, que no podría incluirme en un partido político porque es súper difícil, hay que leer libros sobre eso para poder enterarse de las cosas [...]" (comunicación personal, FG2, s.f.). 


\section{Formación ciudadana deficiente.}

Al evaluar el estado actual de la formación ciudadana, los estudiantes advierten que esta adolece de una serie de falencias, que se identifica -podríamos inferir- a partir de la ya citada postura que asocia indisolublemente la formación política con la participación política, y que a raíz de esta asociación, ve en la formación ciudadana una condición indispensable para la participación en el ámbito de lo instituido.

Por lo mismo es que se depositan expectativas en la educación institucional que, como pudimos constatar, no son satisfechas a cabalidad. Así, tenemos que los informantes establecen que la formación ciudadana se centra sobre todo en la entrega de información, en desmedro de la detención en aspectos reflexivos sobre los mismos contenidos transmitidos. Aquí, los educandos opinan que tras esta forma de transmitir conocimiento existen fines fundamentalmente evaluativos y de cumplimiento con los criterios que establecen los programas escolares, por lo que la profundización, desde ese punto de vista, aparece como innecesaria, como se ilustra en el siguiente fragmento: "Es que se marcó el aspecto más informativo que el de la reflexión, cuando hacen las clases simplemente pasan la materia, para pasar [...] en ese caso era la prueba, entonces, para qué profundizar más $[. .$.$] en ese sentido" (comunica-$ ción personal, FG1, s.f.).

Bastante unido con este último elemento, los jóvenes manifiestan abiertamente la falta de profundización de los contenidos oficiales como una deficiencia de la formación ciudadana, donde se evalúa como negativo el hecho de que el docente centre la transmisión de conocimiento en los contenidos fijados por el Ministerio de Educación:

Siempre se basaban como espina dorsal de todo conocimiento en lo que salía en el libro, no se profundizaba más allá, pero sí en otra materia $[\ldots]$ la historia de Chile era como [...] lo que estaba en el libro (comunicación personal, FG1, s.f.).

En esta misma línea, se percibe que el programa a cumplir por los docentes, es deficiente en términos de profundidad de los contenidos. Así, surge la idea de que la actividad pedagógica, aunque intente ahondar en los contenidos establecidos como básicos por el Estado, no puede hacerlo, puesto que existe un programa al cual debe atenerse, programa cuyo cumplimiento es ciertamente vigilado:

[...] quizás tampoco se puede criticar al profesor, porque a ellos les dan, hasta donde yo sé, un programa que tienen que cumplir, entonces sí, ellos tienen que regirse por eso. No sé si tienen la posibilidad de salirse pero... al menos en el tema del golpe militar, si no se hablaba silenciosamente, no se hablaba [...] y se pasa por encima [...] (comunicación personal, FG1, s.f.).

Así mismo, los estudiantes opinan que la formación ciudadana no apunta a generar nuevos conocimientos, o conocimientos "propios", a partir de los contenidos entregados. Consideran que la educación política termina en la entrega de información, dejando de lado la reflexión a partir de los contenidos, la formación de opinión y elementos como la importancia de la participación política:

Y aparte es como, no sé cómo decirlo, es más, no se genera conocimiento, sino que a nosotros nos dan esos conocimientos, los instalamos y listo, porque, por lo menos nunca en mi caso personal, no recuerdo que me hayan dicho es súper importante que ustedes participen $[. .$.$] (comunicación personal, FG1, s.f.).$

\section{Formación ciudadana sesgada.}

En relación con la misma evaluación que los alumnos realizan sobre las deficiencias de la formación ciudadana actual, corresponde detenerse en la clara inflexión que se realiza 
respecto de la identificación de un sesgo de raíz evidente y eminentemente político. En este sentido, se refieren al peso del golpe de Estado y la posterior dictadura cívico-militar, en la formación ciudadana. Los participantes señalan que a partir de estos hechos histórico-políticos, se ha impedido la enseñanza de educación política, así como de la importancia de la participación política, siendo un hito que afecta la formación política a través de los años posteriores:

[...] si estamos repitiendo tanto el golpe militar, es porque eso es una de las cosas que según yo, no nos ha permitido, después hacia adelante, que nos enseñen de manera clara cómo es nuestra participación [...]. Nos cortaron una parte de que, es como si $[\ldots]$ nos hubieran cortado los pies de nuestro conocimiento cívico, entonces eso viene de ahí hacia adelante y de ahí hacia adelante empiezan a cortar hacia arriba (comunicación personal, FG1, s.f.).

En este punto los estudiantes problematizan la existencia de una censura institucionalizada sobre la profundización en formación ciudadana, ya que indican que los libros de texto del Ministerio de Educación presentan un claro sesgo. Atribuyen esto a la dictadura, por lo que piensan que algún tipo de profundización por parte del profesorado, es entendido como una acción necesaria.

No obstante, dicha profundización está también vetada por las autoridades escolares, con sanciones que pueden llegar al despido de los profesores. Por ello, es que los docentes deben optar por apegarse al programa o realizar algún tipo de profundización en cuanto a formación ciudadana, a espaldas de la autoridad educativa, y con riesgo de ser sancionados: "con la profesora de historia que nosotros teníamos, ella no nos habló más allá, como que profundizó una cosa silenciosa, porque si salía del curso hacia afuera, la profesora era la que tenía que pagar [...]" (comunicación personal FG1, s.f.).

\section{Rol politizador de la formación ciudadana.}

Ya indicamos cómo surge en los discursos la necesidad de la formación ciudadana, así como la ligazón con la participación política, y las consiguientes demandas que se realizan a la formación institucional. En este último punto, queremos destacar una inflexión, donde es explícita que, desde la concepción de los estudiantes, la formación ciudadana debe apuntar a un fin político, que es la participación de los jóvenes en el cambio social.

Como evidencia de esto, los discursos abordan la falta de relevancia de la formación ciudadana en educación como problema a superar. Junto con extrañar la presencia de una asignatura formal, reconocen también como un requerimiento elemental que se enseñe sobre la importancia de la participación de los jóvenes en la sociedad. Sin embargo, al mismo tiempo, se le adjudica a dicha ausencia, la coartada para una "comodidad" no participativa, que delega a otros el ejercicio de la actividad política, actitud que sería contrarrestada justamente con una educación que considerara estos elementos:

Y también la comodidad va... por esa desinformación que dicen los chiquillos, porque a nosotros siempre se nos ha inculcado eso [...] nunca, por lo menos nosotros no teníamos educación cívica cuando estábamos en el colegio y no nos dicen cuál es la importancia de nuestra participación en la sociedad [...] nunca nos han inculcado que esa comodidad no es positiva (comunicación personal, FG1, s.f.).

A su vez, se espera que la formación ciudadana entregue elementos para desarrollar la crítica de las instituciones políticas tradicionales, sobre todo en lo que respecta a la revisión de la formación histórico-política de las mismas (el recuerdo de la contingencia de la formación del orden hegemónico), lo que se relacionaría, según los estudiantes, con una postura más participativa: 
Hace un tiempo [...] vimos esto respecto a lo de las constituciones políticas y yo me lamenté al respecto, me tocó este tema, porque no sabía, que hubo... ha habido tres constituciones políticas y de las tres, nunca se ha hecho como debe ser una Constitución Política, con el real pensamiento de la ciudadanía [...]. Entonces creo que no... que dejaron ajenos al gran porcentaje de la ciudadanía, los dejaron fuera y creo que en este momento no estaría para nada conforme con eso (comunicación personal FG2, s.f.).

Muy ligada con este punto, está la visión de la formación ciudadana como método de concientización de los jóvenes, a propósito de los niveles de desinformación e información deficiente $\mathrm{y} / \mathrm{o}$ sesgada. Otro factor importante en cuanto al rol politizador de la educación, es la concepción de la formación ciudadana como formadora de los futuros liderazgos políticos, puesto que se señala que la enseñanza de la política en la escuela es indispensable para lograr un cambio social y político considerable (como elemento para la construcción de un proceso instituyente), lo que está justamente en las manos de las generaciones que reemplazarán a quienes hoy ocupan los cargos de poder en las instituciones estatales:

Si queremos un cambio en el futuro, en 20, 25 años más vamos a ser nosotros los que vamos a poder estar ahí y no la misma gente que está hoy en día, y yo creo que nosotros, en la educación es fundamental a los alumnos que tengamos les enseñemos política, o les hablemos transversalmente para que puedan dirigir bien el país, porque en el futuro van a ser ellos quienes van a tomar las decisiones [...], entonces no es la idea que la siga dirigiendo la misma gente, nosotros deberíamos ser más responsables y tratar de ejercer algún tipo de presión para lograr un cambio (comunicación personal, FG2, s.f.).

Por último, se establece la figura del docente como agente generador del cambio político-social, a través tanto del traspaso de contenidos como de la entrega de valores, excluyendo a su vez, la búsqueda de cambios por medio de la disputa política con el gobierno de turno.

\section{Vínculo entre formación ciudadana y participación política.}

Esta categoría funciona como una metacategoría, puesto que, de manera más o menos evidente, está presente transversalmente en los discursos de los educandos. Se observa que se realiza una vinculación entre formación ciudadana y participación política, desde que se problematiza la carencia de educación política en la escuela:

Bueno, yo pienso que tenemos poca educación política. Por lo menos, en mi caso jamás me hablaron de partidos políticos ni nada parecido en el colegio, ni en enseñanza media, recién conocí esa situación ingresando a la universidad, por tal, es difícil que yo me defina como hacia algún lado, porque realmente es muy poco lo que conozco (comunicación personal, FG2 s.f.).

Posteriormente, los estudiantes establecen que esta carencia de formación ciudadana se conecta con el desinterés político, y en consecuencia, con la baja participación política (en ambos ámbitos). Es con base en esto, que los alumnos piensan en su formación ciudadana, la evalúan críticamente y generan expectativas respecto de ella, planteándole desafíos y responsabilidades, tal como lo describimos a través de las categorías anteriores. Es así como, a propósito de la problematización del establecimiento de nexos entre formación ciudadana y participación política, los informantes esbozan la necesidad de que los actores políticos tradicionales tomen parte en la educación política:

Pienso que sí necesitamos un cambio, necesitamos más educación política en los colegios, $[\ldots]$ a lo mejor si los partidos políticos se dieran el tiempo de clasificarse, de ir a un colegio, a un liceo, de hablar con los alumnos, de mostrarles lo que quieren y hacia donde 
van, yo creo que se interesarían mucho más las personas, los jóvenes por participar activamente en la política, pero en Chile no se hace, no hay educación política, los profesores no te hablan de política (comunicación personal, FG2 s.f.).

\section{Discusión}

El cambio conceptual que significó el paso de la educación cívica a la formación ciudadana, resulta relevante al menos en la esfera teórica, en tanto la labor en el aula se complejiza, incorporando a la transmisión de contenidos, el desarrollo de habilidades y actitudes "para la vida democrática". Como lo expresamos más arriba, el foco de la formación se desplaza desde la formación de subjetividades para la administración del orden hegemónico instituido, hacia el desarrollo de subjetividades para el cuestionamiento de este orden naturalizado y la potencial construcción de momentos instituyentes. Por cierto que este desplazamiento, viniendo de una institución administrativa, no es radical.

Teniendo en cuenta que la escuela cumple un papel no exclusivo pero sí crucial en la formación ciudadana (Muñoz \& Torres, 2014), es del todo forzoso evaluar este aporte. En esta evaluación, sin lugar a dudas se debe analizar diversos aspectos relacionados, como el marco curricular y el rol docente. Hasta el momento, el examen hecho por diversas investigaciones ha dejado ver el déficit de formación en los estudiantes (Mardones, 2012).

En concordancia con ello, nuestros datos preliminares muestran que la evaluación crítica que ejecutan los jóvenes es más bien negativa. Es probable que las exigencias académicas de la vida universitaria en las áreas investigadas (ciencias sociales) y la interacción con sus pares, les hagan evidenciar una carencia formativa en el ámbito de la política, que no ha sido satisfecha ni en su entorno familiar ni en la escuela. Estos déficits se problematizan como tales en tanto se le plantea un objetivo a la formación ciudadana: generar las condiciones para que exista participación política.

Los participantes evalúan el estado actual de la formación ciudadana como deficiente, en tanto está fundamentalmente centrada en la entrega de información, en detrimento de una reflexión que les permita generar nuevos conocimientos o conocimientos "propios". Este elemento, que requiere mayor exploración, presenta un desafío tanto para los programas educativos, como para las políticas sobre formación docente.

Existe también una clara crítica a la falta de autonomía docente respecto de la formación ciudadana, lo cual llama la atención sobre el alto grado de control que los jóvenes perciben que el Estado y los administradores particulares tienen sobre la formación política. Además, la evaluación negativa que tienen de la formación ciudadana, puede estar poniendo de manifiesto la tensión presente, ante la ya reconocida complejidad de la tarea docente (Muñoz \& Torres, 2014), de efectuar la cobertura de los contenidos y la transposición didáctica que debe realizar para que el estudiante pueda vivenciarlos en el aula.

En consonancia con lo anterior, los participantes en este estudio identifican un sesgo sobre la formación ciudadana actual, el que vinculan al peso de la dictadura cívico-militar. Este sesgo se expresa, según los jóvenes, en la existencia de una censura institucionalizada sobre la profundización en educación política, contenida en los libros de texto ministeriales.

El deficiente abordaje en los textos escolares no es nuevo y ya ha sido pesquisado en algunas investigaciones como la de Minte, Orellana y Tello (2013), que prueban el superficial tratamiento que se da en ellos al contenido ciudadanía, restringiéndolo al sufragio y elecciones. Esto es un problema, ya que, como vimos, los estudiantes le asignan un rol politizador a la educación escolar, donde los profesores 
aparecen como agentes generadores del cambio político-social. Este rol demandado, supone un notable desafío para la labor docente, considerando el material oficial para la formación y las condiciones que limitan el trabajo en el aula.

Pensamos que es en la visualización del vínculo entre formación ciudadana y participación política que se funda la evaluación negativa que hacen los jóvenes de su educación política escolar, lo que a la vez les genera expectativas y demandas respecto de ella. Es destacable, en este sentido, que los jóvenes vean en la formación ciudadana la creación de los futuros liderazgos que asumirán la administración de la política, puesto que indica, contrario al discurso dominante (Urrutia, 2004), que sí existe interés en la juventud por participar políticamente, y más aún, hacerlo en las esferas institucionales, aunque no en la forma en que los partidos tradicionales lo han venido haciendo hasta ahora. Esto puede leerse como un interés por participar en el ámbito de la política y lo político a la vez; esto es, considerando la administración de lo instituido, pero también pensando en la construcción de otros órdenes políticos.

\section{Conclusiones}

Los gobiernos de los últimos años han identificado un descenso en la participación política, que interpretan como un inconveniente de representatividad del sistema político institucional actual. Esta preocupación por el funcionamiento legítimo de las instituciones democrático-liberales, se ha traducido, de acuerdo con Mardones (2012), en análisis poco concretos y autocomplacientes que, en definitiva, no han significado cambios institucionales o curriculares.

Quienes sí realizan un análisis crítico y fundamentado son los estudiantes, quienes ven que las preocupaciones provenientes desde los gobiernos, asociadas a la participación política, resultan poco relevantes, en tanto su expresión práctica más concreta. Es decir, la formación ciudadana en la escuela resulta insuficiente al estar, como diría Retamozo (2009), centrada en la administración de lo ya instituido: la política, y no en la dimensión instituyente en la sociedad: lo político.

Es más, ni siquiera el desarrollo mínimo de lo estipulado por el Ministerio de Educación de la República de Chile, es realizado en el aula, lo que plantea la necesidad no solo de revisar los contenidos del currículo y los planes de formación docente, sino de crear estrategias de participación que involucren en su confección, a los actores más afectados con estos. Por cierto, es igualmente importante generar investigación para producir políticas educativas que mejoren las condiciones de trabajo de los profesores, para este caso, en términos de autonomía en el aula.

Corresponde también comprender la situación histórica y social del fenómeno que se estudia. Aunque aceptáramos la necesidad - planteada desde el Estado- de contar con "ciudadanos comprometidos", ¿cómo se construyen estos, si se tiene un sistema político que no permite la participación efectiva, el cambio político? En efecto, la situación política del país, parece estar lejos del ideario arendtiano de la democracia deliberativa, donde hombres y mujeres en condición de igualdad se reúnen para tratar temas comunes. En su lugar, prima la administración del orden, desde la lógica de la anulación de la contingencia a través de la policía y la política (en su acepción tradicional).

Cabe preguntarse además: ¿qué ciudadanía es la que corresponde formar en los escenarios cultural y político-institucional actuales? Nuestra respuesta es que se debe formar una ciudadanía que tenga las herramientas necesarias para impulsar y trabajar por el cambio social, tanto en lo político-institucional como en lo político-relacional, en el mundo cotidiano, de modo que la contingencia del orden social, se desarrolle dentro de un sistema democrático pleno, y el ámbito 
de lo político tribute justamente a la transformación del orden democrático.

Por lo mismo, es que la formación ciudadana tanto en sus aspectos teóricos como prácticos/ metódicos, debe consistir más que en un conjunto de conocimientos sobre el Estado y sus organizaciones -lo cual no deja de ser vital- en un conjunto de estrategias que permitan a los ciudadanos cuestionar las instituciones que les rodean y las estructuras, tanto concretas como simbólicas, que median sus relaciones interpersonales.

\section{Nota}

${ }^{1}$ La Concertación de Partidos por la Democracia fue una alianza política y electoral, que reunió a sectores del centro y la centroizquierda política chilena y que gobernó el país por cuatro periodos presidenciales consecutivos, entre 1990 y 2010.

\section{Referencias}

Acosta, G., \& Garcés, A. (2010). Ámbitos y escenarios de la participación de participación política juvenil en Medellín. Anagramas. Rumbos y sentidos en la comunicación, 8(16), 15-31.

Arditi, B. (1995). Rastreando lo político. Revista de Estudios Políticos (Nueva Época), 87, 333-351.

Asociación Internacional para la Evaluación del Logro Educativo [IEA]. (2010). Estudio internacional de educación cívica y formación ciudadana ICCS 2009. Chile. Recuperado de www.mineduc.cl

Baño, R. (1985). Lo social y lo político. Un dilema clave del movimiento popular. Santiago de Chile: FLACSO.

Castro-Gómez, S. (2000). Ciencias sociales, violencia epistémica y el problema de la “invención del otro". En E. Lander (Comp.),
La colonialidad del saber: eurocentrismo $y$ ciencias sociales. Perspectivas Latinoamericanas (pp.145-161). Buenos Aires: CLACSO.

Cárdenas, M., Parra, L., Picón, J., Pineda, H., \& Rojas, R. (2007). Las representaciones sociales de la política y la democracia. Última Década, 15(26), 53-78.

Cortina, A. (2001). El Protagonismo de los ciudadanos. Dimensiones de la ciudadanía. En A. Cortina \& J. Conill (Coords.), Educar en la ciudadanía (pp. 13-30). Valencia: Alfons Magnànim.

Díaz, A. (1996). Los primeros de la clase y los últimos románticos. Una etnografía para la crítica de la visión instrumental de la enseñanza. Madrid: Siglo XXI.

Domingo, A. (2001). Ciudadanía multicultural y filosofía política: un desafío cultural al pluralismo. En A. Cortina \& J. Conill (Coords.), Educar en la ciudadanía (pp. 63-83). Valencia: Alfons el Magnànim.

Fernández, G. (2000). Notas sobre la participación política de los jóvenes chilenos. En S. Balardini (Comp.), La participación social y política de los jóvenes en el horizonte del nuevo siglo (pp. 87-108). Buenos Aires: CLACSO.

Garcés, Á. (2010). De organizaciones a colectivos juveniles panorama de la participación política juvenil. Última Década, 18(32), 61-83.

Gómez, J. (2010). Política, democracia y ciudadanía en una sociedad neoliberal, Chile: 1990-2010. Santiago de Chile: ARCIS, PROSPAL, CLACSO.

Instituto Nacional de Juventud [INJUV]. (2003). Cuarta Encuesta Nacional de la Juventud. Santiago de Chile: Autor. 
Instituto Nacional de Juventud [INJUV]. (2010). Sexta Encuesta Nacional de la Juventud. Santiago de Chile: Autor.

Malone, C., \& Julian, G. (2005) Democratic action research (DARE) and large scale simulations: teaching political literacy and civic engagement at Pace University's presidential convention 2004. PS, Political Science \& Politics, 38(4), 771-777.

Mardones, R. (2012). Formación ciudadana, clave de la consolidación democrática. Civilizar, 12(22), 93-110.

Marshall, T. (1998). Ciudadanía y Clases Sociales. Madrid: Biblos.

Ministerio de Educación de la República de Chile [Mineduc]. (2004). Formación ciudadana. Actividades de apoyo para el profesor, Historia y Ciencias Sociales $1^{\circ}$ Básico a $4^{\circ}$ Medio. Santiago de Chile: Mineduc.

Minte, A., Orellana, C., \& Tello, D. (2013). Formación ciudadana en los textos escolares de historia de Chile. Íber. Didáctica de las Ciencias Sociales, Geografia e Historia, 75, 18-24.

Muñoz, C., Vásquez, N., \& Reyes, L. (2010). Percepción del estudiantado de enseñanza básica sobre el rol del Estado, las instituciones públicas, la democracia, la ciudadanía y los derechos de las mujeres y de los inmigrantes. Estudios Pedagógicos, 36(2), 153-175.

Muñoz, C., \& Torres, B. (2014). La formación ciudadana en la escuela: Problemas y desafíos. Revista electrónica Educare, 18(2), 233-245.

Navarrete, B. (2008) Juventud y política en liceos municipales. El caso de Maipú. Última Década, 16(28), 167-202.
Navia, P. (2004). Las grandes alamedas. El Chile Post Pinochet. Santiago de Chile: La Tercera-Mondadori.

Rajland, B. (2003). Crisis de Hegemonía, crisis de representación. La articulación de lo social y lo político. Buenos Aires: CEFJA.

Reguillo, R. (2000). Emergencia de culturas juveniles. Estrategias del desencanto. Bogotá: Norma.

Retamozo, M. (2009). Lo político y la política: los sujetos políticos, conformación y disputa por el orden social. Revista Mexicana de Ciencias Politicas y Sociales, 51(26), 69-91.

Rojas, E. (2006). Los jóvenes y el plebiscito de 1988: Aproximación a las motivaciones de los jóvenes por participar del plebiscito de 1988. Informe de Seminario para optar al grado de licenciado en Historia. Santiago: Universidad de Chile.

Sandoval, J., \& Hatibovic, F. (2010). Socialización política y juventud: el caso de las trayectorias ciudadanas de los estudiantes universitarios de la región de Valparaíso. Última Década, 18(32), 11-36.

Serna, L. (2000). Las organizaciones juveniles. De los movimientos sociales a la autogestión. Jóvenes, 4(11), 114-130.

Urresti, M. (2000). Paradigmas de participación juvenil: un balance histórico. $L a$ participación social y la política de los jóvenes en el horizonte del nuevo siglo. Buenos Aires: CLACSO.

Urrutia, M. (2004) La hégira Touraine y el perpetuo ocaso de los movimientos sociales en América Latina. Ciencias Sociales Online, 3(1), 64-75. 
Valenzuela, K. (2007) Colectivos juveniles. ¿Inmadurez política o afirmación de otras políticas posibles?. Última Década, 15(26), 31-52.

Vargas, C. (2005). El Género y la participación Política en Puerto Rico. Revista Caribbean Studies, 33(1), 205-248.

Zarzuri, R. (2005). Jóvenes, participación y movimientos sociales: hacia la construcción de nuevas formas de participación juvenil. Santiago de Chile: Centro de Estudios Socioculturales. 
